\title{
CAN IMPAIRED MOTOR AND COGNITIVE FUNCTIONS AT 10 YEARS OF AGE BE IDENTIFIED BY A DETAILED ANALYSIS OF INFANT MOTOR BEHAVIOUR IN VERY-LOW- BIRTH-WEIGHT CHILDREN?
}

\author{
T. Fjørtoft ${ }^{1}$, K.H. Grunewaldt ${ }^{2,3}$, T. Ustad ${ }^{1}$, G.C.C. Løhaugen ${ }^{2,4}$, J. Skranes ${ }^{2,3,4}$, K.A.I. Evensen ${ }^{1,5}$ \\ ${ }^{I}$ Dept of Clinical Services, St.Olavs Hospital, Trondheim University Hospital, ${ }^{2}$ Lab. Medicine, Children's \\ and Women's Health, Norwegian University of Science and Technology, ${ }^{3}$ Pediatrics, St. Olavs Hospital, \\ Trondheim University Hospital, Trondheim, ${ }^{4}$ Pediatrics, Sørlandet Hospital, Arendal, ${ }^{5}$ Public Health and \\ General Practice, Norwegian University of Science and Technology, Trondheim, Norway
}

Background: The General Movement Assessment has mainly been used to identify children with cerebral palsy (CP). A more detailed analysis of infant motor behavior can possibly identify later motor and cognitive problems in children without $\mathrm{CP}$.

Aims: To determine whether such a detailed analysis of infant motor behaviour can predict motor and cognitive development in very-low-birth-weight children at age 10 .

Methods: Twenty-two infants without CP with mean gestational age 26.9 (SD2.0) wks; mean birth weight 888 grams (SD229) were included. Video-recordings were analyzed according to "Assessment of Motor Repertoire 3-5 Months", which is part of Prechtl's General Movement Assessment. Fidgety movements were classified as present or absent/abnormal. Quality of the whole motor repertoire was classified as normal if smooth and fluent and abnormal if jerky, monotonous or stiff. At age 10, poor outcome was defined as Movement Assessment Battery for Children-2 (MABC-2) score below the $5^{\text {th }}$ percentile or a total IQ less than 85 on the Wechsler Intelligence Scale for Children (WISC-III).

Results: Most children with poor motor outcome were identified by presence of fidgety, but abnormal motor repertoire, at 14 weeks post term (sensitivity: $0.83 ; 95 \%$ CI: $0.44-0.97$ ). Sensitivity of presence of fidgety, but abnormal motor repertoire, for poor cognitive outcome was 0.50 (95\%CI: $0.15-0.85$ ). Specificity was 0.75 (95\%CI: 0.51-0.90) and 0.61 (95\%CI: 0.39-0.80) for normal motor and cognitive outcome, respectively.

Conclusions: Presence of fidgety movements, but abnormal motor repertoire, may predict later impaired motor and to a lesser degree cognitive development in preterm children who do not develop CP. 